\title{
Luminescence of phosphorus doped silica glass
}

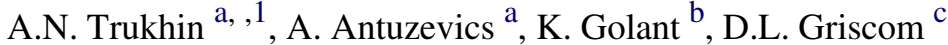 \\ ${ }^{a}$ Institute of Solid State Physics, University of Latvia, LV-1063 Riga, Latvia \\ ${ }^{b}$ Institute of Radio-engineering and Electronics, RAS, 125009 Moscow, Russia \\ ${ }^{\mathrm{c}}$ Impact Glass Research International, 3938 E Grant Rd \#331, Tucson, AZ 85712, USA
}

\begin{abstract}
a b s tract
A fiber preform with P-doped silica core is studied by luminescence methods. P-doped silica was synthesized via the SPCVD method on a substrate tube made of pure silica glass F300. Two luminescence bands were detected under excitation of the $F_{2}$ excimer laser $(157 \mathrm{~nm})$. One band is in UV range at $4.6 \mathrm{eV}(265 \mathrm{~nm})$ with two time con-stants $\sim 30 \mathrm{~ns}$ and $5 \mu$ s and the other at $3.1 \mathrm{eV}(400 \mathrm{~nm})$ with time constant $\sim 5.5 \mathrm{~ms}$. Fast decay of the blue band with time constant $\sim 20 \mathrm{~ns}$ was also observed. The main excitation band of the UV luminescence is at $7.1 \mathrm{eV}(\sim 170 \mathrm{~nm})$ and that for blue band is at $6.3 \mathrm{eV}(\sim 195 \mathrm{~nm})$. These bands belong to two different luminescence centers, however, both are associated with the presence of phosphorus. The UV band is similar to the one observed in many different oxide materials containing phosphorus and is ascribed to $\mathrm{PO}_{4}^{3}{ }^{-}$complex ion. The blue band is as-cribed to a twofold coordinated phosphorus. Both the blue and the UV luminescences participate in the recom-bination process due to electron trapping. These luminescences appear due to thermal stimulation upon recombination of liberated self-trapped holes. Other than the detected phosphorus-related oxygen-hole-centers, there is no other recombination luminescence.
\end{abstract}

\section{Introduction}

Phosphorus is one of the dopants in silica glass changing the index of refraction in order to adjust the properties of optical fibers. On the other hand, phosphorus is interesting for codoping ions in many materials, thus useful for fundamental research. Phosphorus takes on a variety of valence states in different oxides doped with phosphorus. The phos-phite ion $\mathrm{PO}_{3}^{3}{ }^{-}$, the orthophosphate ion $\mathrm{PO}^{3} 4^{-}$, and the pyrophosphate ion $\mathrm{P}_{2} \mathrm{O}^{4} 7^{-}$are among them. Also intriguing could be phosphorus mon-oxide, as well as the $\mathrm{PO}_{2}$. These ions determine various phenomena in those oxides. It is found that the $\mathrm{PO}^{3} 4^{-}$complex is the main structural el-ement in silicon dioxide doped with phosphorus [1].

Phosphorus gives its name to one kind of luminescence - phosphorescence - thus it is natural to study properties of phosphorus doped materials by luminescence methods.

Analyses of literature related to the luminescence of different mate-rials with phosphorus (e.g. $[2,3]$. show that the common luminescence property of these materials is the observation of a UV band. Thus, in $\mathrm{ScPO}_{4}$ zircon-type crystal the band at $5.7 \mathrm{eV}$ is interpreted as self-trapped exciton (STE) of this crystal [2]. $\mathrm{AlPO}_{4}$ and $\mathrm{GaPO}_{4}$, both with structure of a-quartz, give rise to the $\mathrm{UV}$ bands situated at $5.83 \mathrm{eV}$ and $5.65 \mathrm{eV}$ respectively, and are thus interpreted as the second STE related to orthophosphate ions [2]. The first STE is similar to that of $\alpha$-quartz, which band is situated in the visible range of the spectrum. In phosphorusdoped crystalline $\alpha$-quartz, phosphor-silicate glass, and lime phosphate glasses, the UV bands are situated at $4.9 \mathrm{eV} ; 4.8 \mathrm{eV} ; 4.1 \mathrm{eV}$ respectively [3] These bands are interpreted as luminescence of phosphorus related defects in the corresponding structure. In all the cases the UV band exhibits relatively fast decay in ns range of time, as-cribed to singlet-singlet transitions with decay times of tens of $\mu$ s due to triplet-singlet transitions. A model for the UV luminescence center is assumed on the basis of the STE in ScPO4, where the hole component is situated on orthophosphate and the electron component is dispersed around scandium. Mentioned materials have differing in properties. $\mathrm{ScPO}_{4}$ is a dry crystal, whereas a phosphorus-doped crystalline quartz sample was found to contain about 3400 atomic ppm of water and $\mathrm{OH}$ groups. However, the existence of UV luminescence does not depend on water content.

Another situation is the non-UV band luminescence in the men-tioned materials. In $\mathrm{ScPO}_{4}$, huge amounts of different luminescence bands were observed (see e.g. [4]), mainly related to impurities.

The luminescence of phosphorus doped crystalline quartz sample exhibits yellow-red bands at $600 \mathrm{~nm}(2.065 \mathrm{eV})$ and $740 \mathrm{~nm}(1.65 \mathrm{eV})$. Corresponding centers are created by excitation light. A sim-ilar yellow luminescence was observed for $3 \mathrm{P}_{2} \mathrm{O}_{5} \cdot 7 \mathrm{SiO}_{2}$ glass [3]. How-ever, in Ref. [5] silica glass fiber preforms with phosphorus in the core do not exhibit such yellow luminescence. Instead, there is a blue lumi-nescence with a time constant of about $6 \mathrm{~ms}$ was observed. It is 
necessary to underline that this blue luminescence was mainly ob-served under excitation with a UV laser. In Ref. [5], there was also an at-tempt to measure photoluminescence excitation spectra excited by synchrotron light. However, observation was made on a total slice of preform including the cladding. So, it is not certain that the excitation spectrum corresponded to Pdoped core.

So, in this work we have performed studies of luminescence in phosphorus-doped silica glass obtained by chemical vapor deposition (Sur-face Plasma Chemical Vapor Deposition). Material obtained through this method contains very little water or $\mathrm{OH}$ groups. In that regard, it is different from previously studied materials [3]. As for previous sam-ples, we have compared a UV luminescence with similar properties. However, yellow-red luminescence was not observed at all. Instead, we observed blue luminescence with long duration kinetics, as was an-tecedently discovered in [5].

Blue luminescence related to phosphorus was observed in KCl-P crystals [6]. It is remarkable that the slow decay (a few $\mathrm{ms}$ ) of this blue luminescence has been observed, as in the case of Ref. [6]. $\mathrm{PO}^{-} 2$ is responsible for blue luminescence in KCl-P crystal. Finally, we came to the conclusion that similar defects could be responsible for blue lumi-nescence in silica glass with phosphorus. However, in Ref. [5] the blue luminescence was ascribed to a defect consisting of diamagnetic four-coordinated $\mathrm{P}$ impurity substitutional for a $\mathrm{Si}$ atom.

\section{Experimental procedure}

The SPCVD sample was a fiber preform $10 \mathrm{~mm}$ in diameter. The pure silica glass (Suprasil F300) tube was used for deposition of phosphorus containing pure silicon dioxide (see Ref. [7]). After deposition the tube was compressed at high temperature and two preforms with P-doped core were obtained. The concentration was about $1 \%$ of $\mathrm{P}_{2} \mathrm{O}_{5}$. One core was $4 \mathrm{~mm}$ in diameter and the other was $2 \mathrm{~mm}$. Slices of different thick-ness were made: $0.4 \mathrm{~mm}, 1 \mathrm{~mm}$ and $5 \mathrm{~mm}$. Diaphragms were used that avoid excitation of cladding, which were made from pure silica. The pro-file of the index of the preform is presented in Fig.1.

The photon counting mode instrumented with the Hamamatsu H8259-02 module was used for slow luminescence detection. Inside the module beside the PM there were an amplifier, comparator, and a pulse former. This mode was used for low intensity luminescence. Fast intensive luminescence was detected by PMT Hamamatsu H6780-04 in current regime. There PM anode is loaded with a $50 \mathrm{Ohm}$ resistor. Lu-minescence decay curves were recorded by a Tektronics TDS 2022B os-cilloscope, each curve being averaged over 128 pulses. The time-resolved luminescence spectra were extracted from the decay curves measured at each specific wavelength. The corresponding decay curve was integrated within a measured time interval. The $\operatorname{ArF}(193 \mathrm{~nm}$

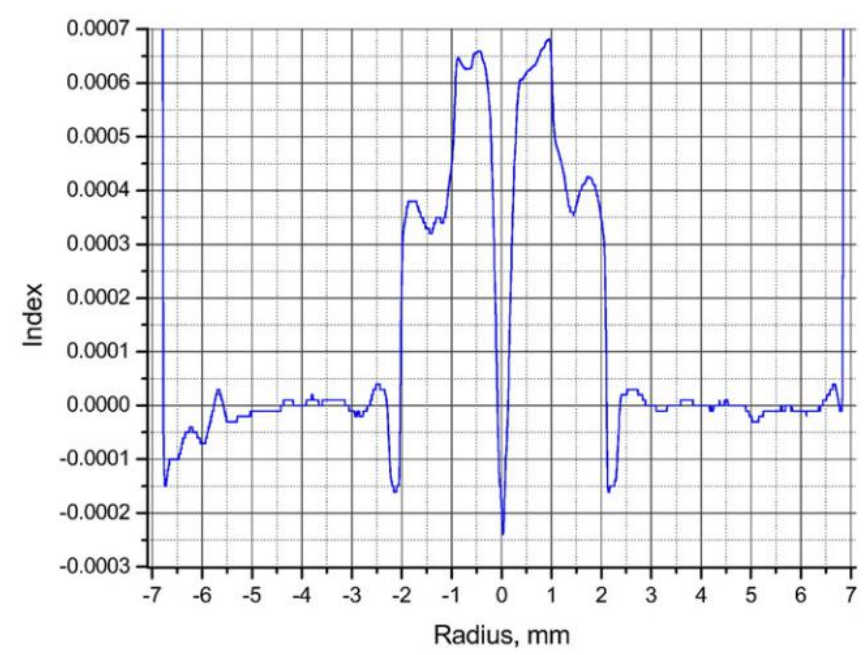

Fig. 1. Profile of index of the preform $\mathrm{P}_{2} \mathrm{O}_{5}: \mathrm{SiO}_{2} 1 \mathrm{~mol} \%-\quad \mathrm{n} \sim 0.001$. wavelength) and $\mathrm{KrF}$ (248 nm wavelength) excimer lasers, model PSX-100 of Neweks (Estonia) with pulse energy of up to $5 \mathrm{~mJ}$ and pulse duration of $5 \mathrm{~ns}$ were used to excite the luminescence. Also, a ten-times less powerful $F_{2}$ laser $(157 \mathrm{~nm})$ was used. Luminescence emission was collected in a direction perpendicular to the excitation laser beam. The samples were carefully cleaned and mounted on a hold-er; no glue was used. Measurements were performed at 60-350 K sam-ple temperatures. The lower temperature of $60 \mathrm{~K}$ was obtained by pumping of liquid nitrogen from cryostat. Some measurements were performed on a helium refrigerator in the range of temperature 10-290 K. The high temperature range was checked in a sample holder with heating.

Luminescence was detected with the help of a grating monochroma-tor (MCD-1) with slit width of about $1 \mathrm{~mm}$ corresponding to $1.5 \mathrm{~nm}$ spectral resolution. The measured curves are mainly presented in the figures as recorded; therefore, they reflect the level of errors. Very noisy curves were smoothed by a sliding average approach. Also, the life time of the gas in the excimer laser was short, with intensity deteri-orating with time. However, frequent changing of the gas is costly, so we tried to perform measurement in time when detectable luminescence could be recorded. Then comparison of intensities within one figure is guaranteed, whereas long-time correspondence related to different pre-sented figures was difficult.

\section{Results}

\subsection{Optical properties}

The optical absorption spectrum of the P-doped core of preform is presented in Fig.2. When measuring a $4 \mathrm{~mm}$ thick sample we detected well resolved bands at $\sim 6.8$ to $7.0 \mathrm{eV}$ and at $\sim 7.3$ to $7.5 \mathrm{eV}$. The optical gap is situated at $8.1-8.2 \mathrm{eV}$ and it is well correspondent with known properties of pure silica position of the optical gap (see e.g. [8]). A $0.4 \mathrm{~mm}$ thick sample was used for analyses of the absorption spectrum near optical gap, Fig.2 insert. For pure silica, the optical gap obeys the Urbach rule, explained by Toyozawa [9] as a momentary self-trapped exciton (STE) creation similar to that of $\alpha$-quartz crystal. The properties of the pure silica optical gap can be found in $[8,10]$. A remarkable prop-erty is the crossing of all spectra in semi$\log$ scale at one point. However, for phosphorus-doped core silica, the obtained spectra for different temperatures on a semi-log scale exhibit parallel shifts with tempera-ture, Fig.2 insert. This case is known for disordered materials. The

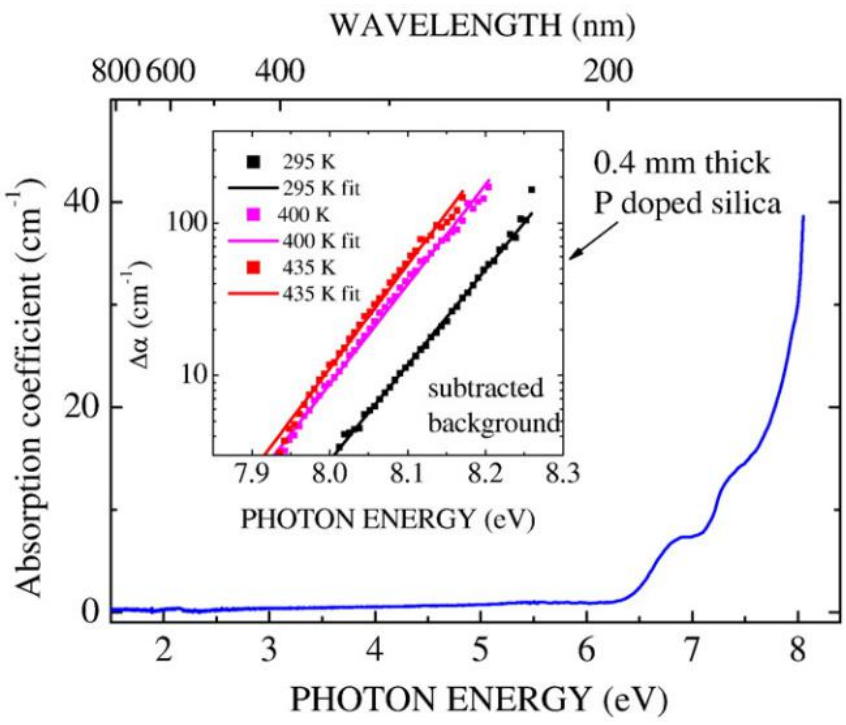

Fig. 2. Optical absorption spectra of silica preform with P-doped core, $4 \mathrm{~mm}$ thick sample Insert - absorption spectra at different temperatures for $0.4 \mathrm{~mm}$ thin sample (points). Lines - fit to expression (1) of glass-like Urbach rule for optical gap. 
intrinsic absorption obeys the Urbach rule in the usual form for disor-dered materials [11,12]:

$\alpha^{1 / 4}$ Io $\exp 1 / 2 \mathrm{AE}$ p $\mathrm{T}=\mathrm{T}_{1} \&$ ð1p

where $\mathrm{A}$ is a parameter of the slope, $\mathrm{T}_{1}$ is a characteristic temperature, and $\mathrm{E}$ is the photon energy and $\mathrm{I}_{\mathrm{O}}$ is fitting parameter. The slope coeffi-cient $\mathrm{A}=15$ $\pm 1 \mathrm{eV}^{-1}$ and the characteristic temperature $\mathrm{T}_{1}=18 \pm$

$3 \mathrm{~K}$ are determined for the case of the P-doped-core sample as $\mathrm{I}_{\mathrm{O}}=$ $5.1 \cdot 10^{-60} \mathrm{~cm}^{-1}$ or $\left(\mathrm{I}_{\mathrm{O}}=\exp (-138.9)\right)$.

Optical absorption of a 5-mm-thick sample is presented in Fig.3a. There are several bands in the core. After irradiation of the core subject-ed to ArF laser light, the band at $2.24 \mathrm{eV}$ increases. In Fig. 3b the absorp-tion spectrum of the P-doped core of the fiber preform is compared with the photoluminescence excitation spectra. However, the PL excitation takes place in the range of absorption bands that are not in direct corre-spondence with the excitation band maxima. Perhaps those absorption bands are not resolved on the background. Moreover, the intensity of photoluminescence excited through vacuum monochromator with low power deuterium discharge source is low and the PLE curves are noisy. The presented PLE spectra are smoothed by a sliding-least-squares approach. The PL spectra are measurable more precisely with excitation by excimer lasers, which do not need smoothing for presen-tation. The photoluminescence bands measured under laser excitation are also presented in Fig.3b. The photoluminescence and its time con-stants for the spectra of a P-doped-core silica fiber preform detected in different ranges of time are presented in Fig.4. There is also a band
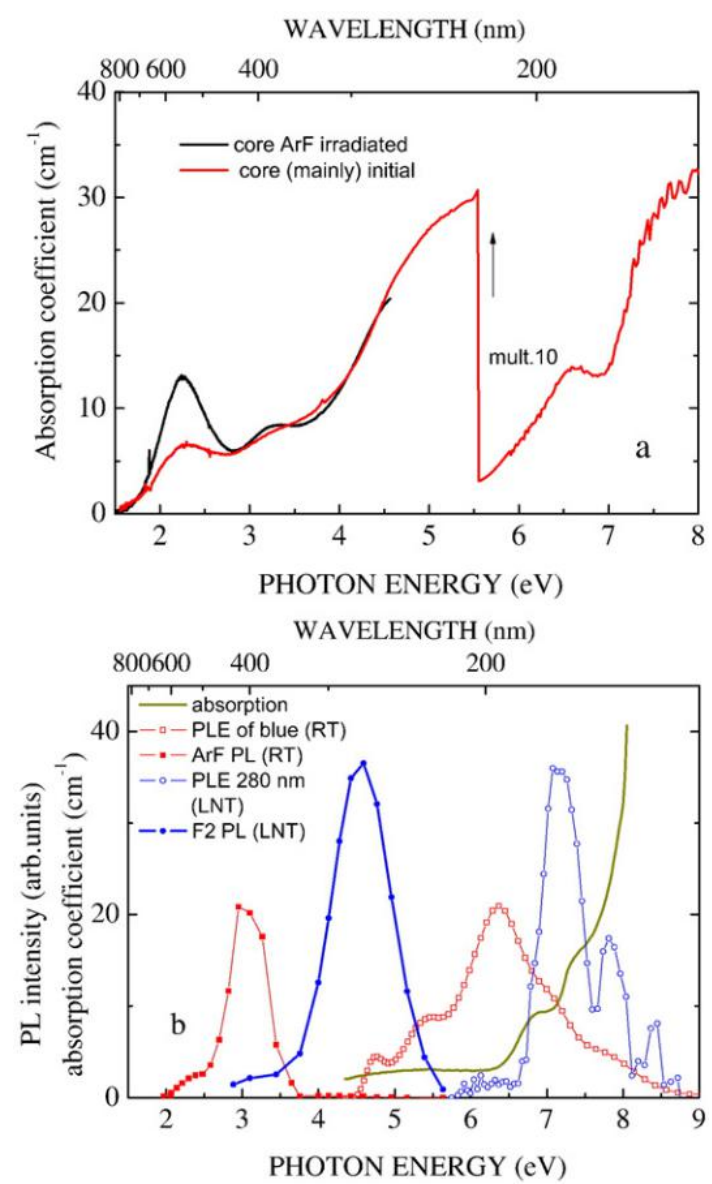

Fig. 3. (a) Optical absorption of $5 \mathrm{~mm}$ sample of silica preform with P-doped core. Part below $5.5 \mathrm{eV}$ is increased 10 times. Irradiation of core with ArF laser provides growth of a band at $2.24 \mathrm{eV}$. (b) Comparison of optical absorption spectrum with photoluminescence excitation spectra (PLE). Smoothing of PLE spectra by sliding least-squares approach was made. The PL bands measured under excimer lasers excitation are presented.

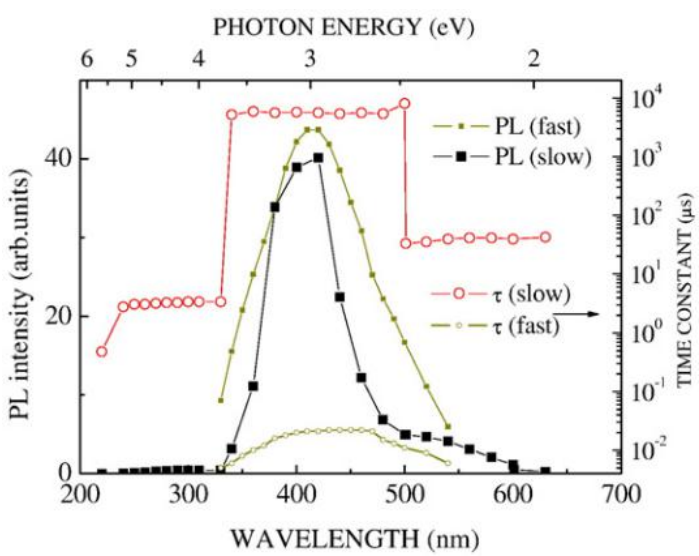

Fig. 4. PL (square) and T (circles) spectra of P-doped silica ArF, $293 \mathrm{~K}$. Small points for fast decay, big points for slow decay. The lines are to guide the eye.

at $400 \mathrm{~nm}$ with time constant $5.2 \mathrm{~ms}$ and smaller band at $600 \mathrm{~nm}$ with a time constant about $40 \mu \mathrm{s}$ (Fig.4). The band at $400 \mathrm{~nm}$ also pos-sesses a fast decay $\sim 20 \mathrm{~ns}$. The corresponding PL band is a bit broader than the band with slow decay. There is also the same PL band at $300 \mathrm{~nm}$ with a decay time in the $\mu \mathrm{s}$ range. It is seen that the band at $400 \mathrm{~nm}$ possesses an almost uniformly distribution of time constants from $5 \mathrm{~ms}$ to $6 \mathrm{~ms}$. The decay kinetics is an exponential (which is rare for glassy materials) after the fast peak seen in Fig.5. The decay duration is not changed with cooling. The intensity of the band at $400 \mathrm{~nm}$ also changes little with cooling from $293 \mathrm{~K}$ to $80 \mathrm{~K}$ Fig.5. Also in Fig. 5, we have studied the polarization properties of the timeresolved blue lumi-nescence of the $\mathrm{P}$-doped core under excitation with $\mathrm{KrF}$ laser through a polarizer made from three gold coated mirrors. The polarization degree was determined as $\mathrm{P}=(\mathrm{V} \backslash \backslash \mathrm{H}) /(\mathrm{V}+\mathrm{H})$, where $\mathrm{V}$ is intensity of lumi-nescence for analyzer parallel to electric vector and $\mathrm{H}$ is perpendicular to that. We found that the blue luminescence is negatively polarized with respect to excitation electric vector direction. The degree of polar-ization is practically independent on temperature. All curves of decay in Fig.5 are normalized to the maximum in initial stage of decay, and their difference does not show a slow luminescence intensity thermal depen-dence. As mentioned above, the intensity changes little with cooling.

In the high temperature range, Fig.6, the intensity of the blue luminescence and the decay time constant possess similar temperature dependences. It's PL I(T) and $\mathrm{T}(\mathrm{T})$ approximate Mott's law [I or $\mathrm{T}=\mathrm{I}_{0}$ or $\mathrm{T}_{0} /(1$ $\left.\left.+\mathrm{f} \cdot \mathrm{T}_{0} \exp (-\mathrm{E} / \mathrm{kT})\right)\right]$, thus providing thermal quenching activa-tion energy $\mathrm{E}$ $=0.5 \pm 0.05 \mathrm{eV}$ and frequency factor $\mathrm{f} \sim 10^{9} \mathrm{~s}^{-1}$.

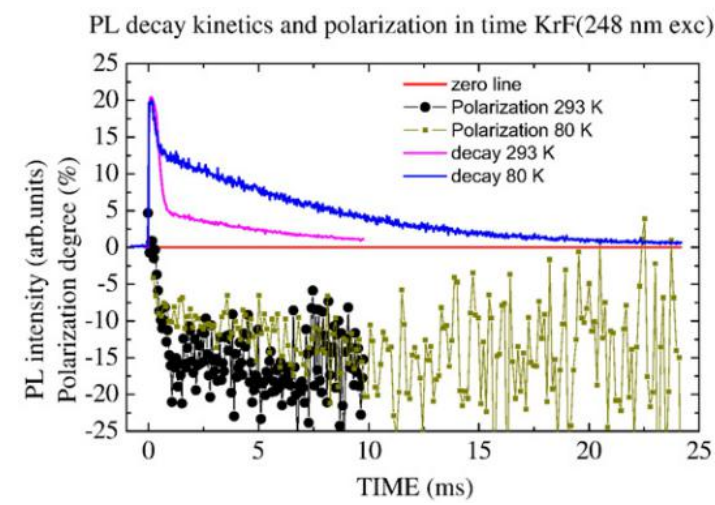

Fig. 5. The example of decay kinetics obtained under $\mathrm{KrF}$ laser. Time resolved blue luminescence polarization determined as $\mathrm{P}=[(\mathrm{V} \backslash \mathrm{H}) /(\mathrm{V}+\mathrm{H})] \cdot 100, \mathrm{~V}-$ decay kinetics for parallel and $\mathrm{H}-$ for perpendicular analyzer with respect to excitation electric vector. So, luminescence electric vector is oriented orthogonally to excitation electric vector. 
Phosphorus doped silica $400 \mathrm{~nm}$ I (T) comparison with ESR

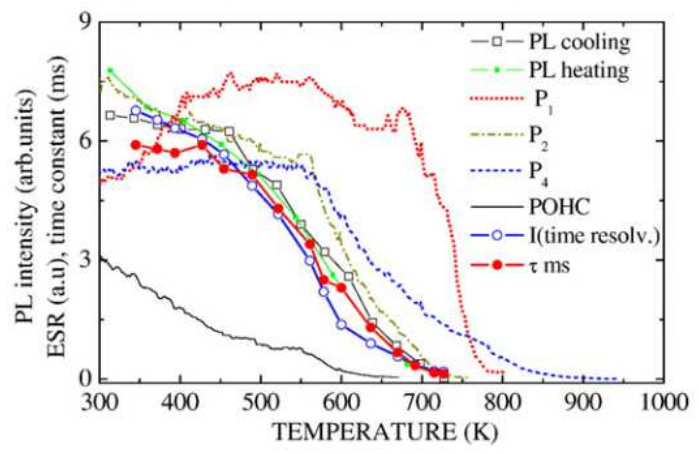

Fig. 6. Thermal dependences of $400 \mathrm{~nm}$ PL, T and I, of phosphorus doped silica under ArF excitation. Points connected by straight lines. PL I(T) and $\mathrm{T}(\mathrm{T})$ approximation with Mott's law [I or $\mathrm{T}=\mathrm{I}_{0}$ or $\left.\mathrm{T}_{0} /\left(1+\mathrm{f} \cdot \mathrm{T}_{0} \exp (-\mathrm{E} / \mathrm{kT})\right)\right]$ provide thermal quenching activation energy $\mathrm{E}=0.5 \pm$ $0.05 \mathrm{eV}$ and frequency factor $\mathrm{f} \sim 10^{9} \mathrm{~s}^{-1}$. The curves marked $\mathrm{P}_{1}, \mathrm{P}_{2}, \mathrm{P}_{4}$ and POHC are ESR data from [1] presented for comparison.

Therefore, these two values are determined by intra center quenching processes.

Although PL is the principle purpose of this paper, Fig. 6 actually represents the number density of radiation-induced phosphorous defects created at $300 \mathrm{~K}$ and subsequently annealed as functions of increasing temperature. Here, thermal quenching of the PL parameter dips from $300 \mathrm{~K}$ to virtually nothing at $750 \mathrm{~K}$ but it can be reversed without loss upon cooling. In Fig. 6 the $\mathrm{P}_{1}, \mathrm{P}_{2}, \mathrm{P}_{4}$, and $\mathrm{POHCs}$ defects are recorded in the range 300 to $750 \mathrm{~K}$. These data employ the same nomenclature as that evolved in fig. 12 of Ref. [1]. However, the latter was measured from $100 \mathrm{~K}$ upward, rather than from $300 \mathrm{~K}$, and it extends to $1150 \mathrm{~K}$. But for present purposes, the data of fig. 12 of Ref. [1] will be truncated at $560 \mathrm{~K}$. So, from $100 \mathrm{~K}$ to $560 \mathrm{~K}, \mathrm{P}_{2}$ is dominant and decreases only $\sim 8 \%$ upon reaching $560 \mathrm{~K}$. $\mathrm{P}_{2}$ is known to be a four-coordinated phosphorus atom that has trapped an electron. Thus, all of the rest are trapped hole centers. $\mathrm{P}_{4}$ is found to move nearly horizontally as the temperature rises from 100 to $560 \mathrm{~K}$. In contrast, $\mathrm{P}_{1}$ grew linearly before flatting out at

$\sim 400 \mathrm{~K}$, and in the same range, the POHC moved downward at the a slightly increasing rate after crossing $\mathrm{P}_{1}$ at $200 \mathrm{~K}$.

In addition, Ref. [1] reported a defect that appeared close to $\mathrm{P}_{1}$ at $100 \mathrm{~K}$, but from there dived steeply downward nearly two orders of magnitude by $300 \mathrm{~K}$. This feature was noted by the authors of [1], who finally decided that it was a type of E' center which they dubbed "Si E'

$(\mathrm{P})$ " because of its narrowness and lack of hyperfine doublets, as

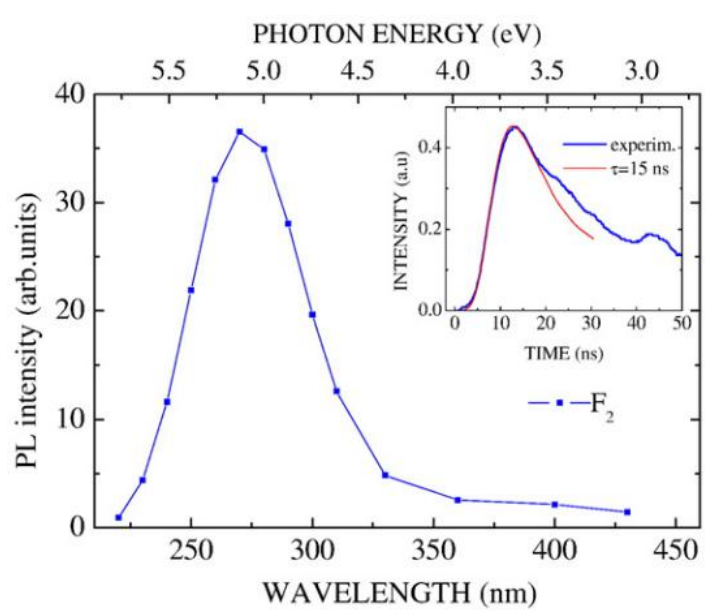

Fig. 7. Time resolved (ns) PL spectra and, insert, decay kinetics of UV band under F2 laser. Time constant $\mathrm{T}$ was determined by fastest fit of laser pulse convoluted with exponent with time constant $15 \mathrm{~ns}$. Discrepancy in higher time shows on existence also of component of decay with higher time constant $\mathrm{T}-80 \mathrm{~K}$

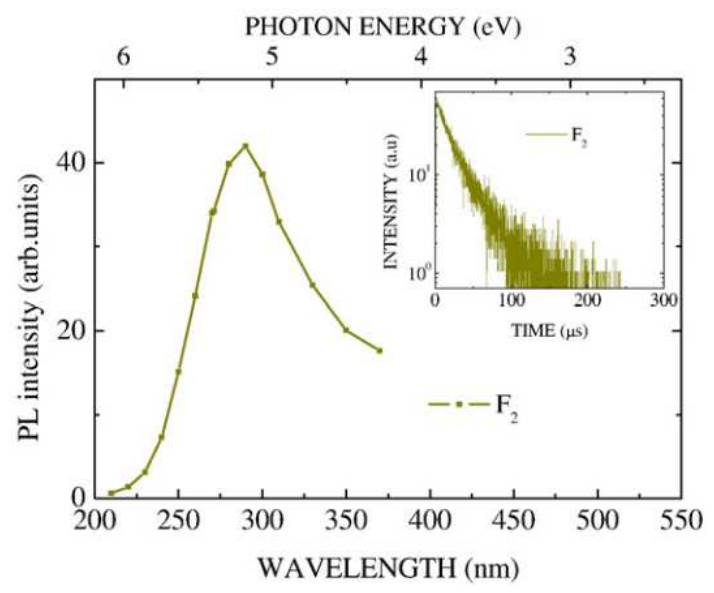

Fig. 8. Time resolved PL spectra in the range of $100 \mu \mathrm{s}$. Insert presents decay kinetics of UV band under $\mathrm{F}_{2}$ laser.

would be expected for phosphorous bearing entities. However, 7 years later Griscom discovered self-trapped holes in silica glass [13], later proved it in detail [14], and finally added additional thoughts in $[15,16]$. In [15] it is found that solid evidence that the "Si E' (P)" found at the low temperature end of $\mathrm{x}$-irradiated $\mathrm{P}_{2} \mathrm{O} 5$ - $\mathrm{SiO} 2 \mathrm{~K}$ [1] had nothing at all to do with phosphorus; rather it was the work of self-trapped holes!

The time resolved PL spectrum in Fig.7 (main part) and decay kinet-ics (insert) of the UV band are due to $F_{2}$ laser excitation. The data of Fig.7 were recorded with the PM in the current regime. In the insert to Fig. 7, an exponential approximation reveals the main decay time constant of $15 \mathrm{~ns}$. Evidently the decay is non-exponential and there is a long dura-tion time as seen in the insert. In Fig.8 the time resolved PL spectrum ex-cited with $\mathrm{F}_{2}$ laser light is presented. The intensities at different points are determined by integration of the decay curves measured with a pho-ton-counting method. An example of this curve is presented in the Fig.8, insert. The duration was about $100 \mu \mathrm{s}$.

In Fig. 9 the UV PL spectra are compared with both the ns and $\mu$ s time ranges under $F_{2}$ laser at $77 \mathrm{~K}$. The shift of the slow duration PL band at the lower photon energy with respect to the fast duration PL band should be noted. For this reason we interpret it as a manifestation of sin-glet-triplet splitting. Therefore, we ascribe the slow UV band to triplet-singlet transitions and the fast UV band to singlet-singlet transitions of

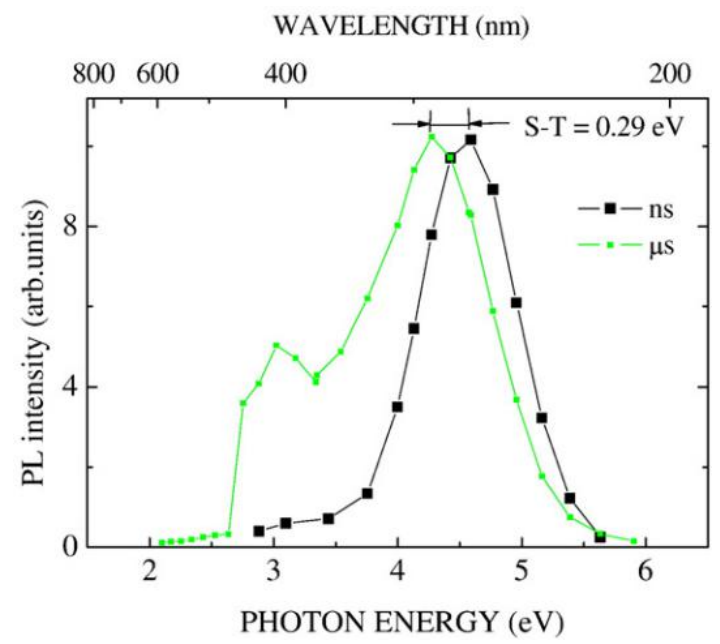

Fig. 9. Comparison of UV PL spectra of P-doped silica preform core measured in the ns and hundreds of $\mu$ s time range under $\mathrm{F}_{2}$ laser at $77 \mathrm{~K}$. Mutual shifts of the bands on $0.29 \mathrm{eV}$ could witness on singlet-triplet splitting of excited state of the luminescence center. 


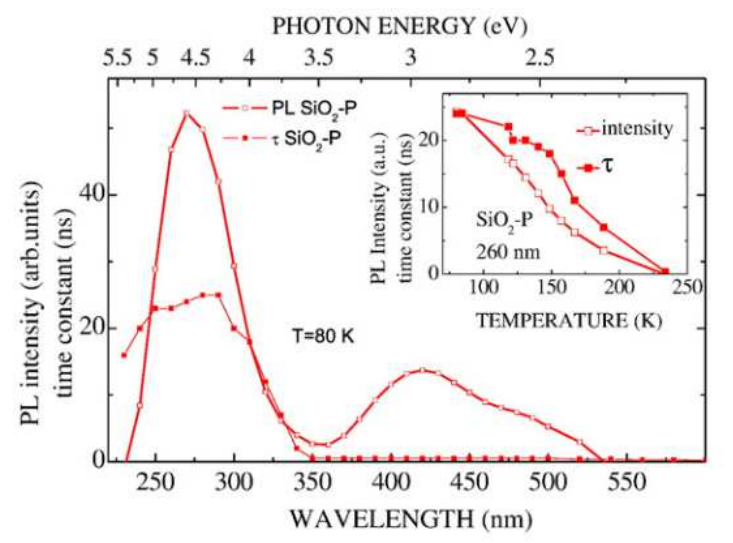

Fig. 10. Time resolved $\mathrm{PL}$ and time constant spectra of $\mathrm{SiO}_{2}-\mathrm{P}$ under $\mathrm{F}_{2}$ laser. Insert PL intensity and time constant temperature dependence for $260 \mathrm{~nm}$ band. Time constant $\mathrm{T}$ was determined analogously to Fig.8, however longest duration was taken. Intensity thermal dependence exhibits exponential growth with cooling usual for disordered materials.

$P$ related luminescence center. The shoulder at $3 \mathrm{eV}$ is evidently a blue band, the data for which are presented above.

The spectral distribution of the decay kinetics of the UV lumines-cence of $\mathrm{SiO} 2-\mathrm{P}$ glass is provided in Fig. 10 for both $\mathrm{PL} \mathrm{SiO} 2-\mathrm{P}$ and T SiO2-P as functions of both wavelength (main figure) and temperature (insert). Of extreme interest is the fact that the lower of the two curves of the insert matches very well $\mathrm{STH}_{2}$ in that range while the higher curve of the insert matches well $\mathrm{STH}_{1}$, as clearly shown in fig. 7 of [15].

\subsection{Radiation properties}

In Fig.11 the luminescence spectra for $\mathrm{x}$-ray and $\mathrm{F}_{2}$ laser excitation are compared. The UV band could also be excited in the thermally stim-ulated luminescence (TSL) regime, insert Fig.11. TSL contains several peaks in the range $100-200 \mathrm{~K}$. Also, the band at $400 \mathrm{~nm}$ could be seen in TSL with a different shape, i.e., having one main peak at $120 \mathrm{~K}$. How-ever, the band at $270 \mathrm{~nm}$ (the green curve) does not behave as TSL upon ArF laser excitation, heading downward, whereas the TSLs all started at zero and headed up. Indeed, during steady state excitation with x-rays, the intensity of the band at $270 \mathrm{~nm}$ increases with cooling, Fig.11, I(T) insert.

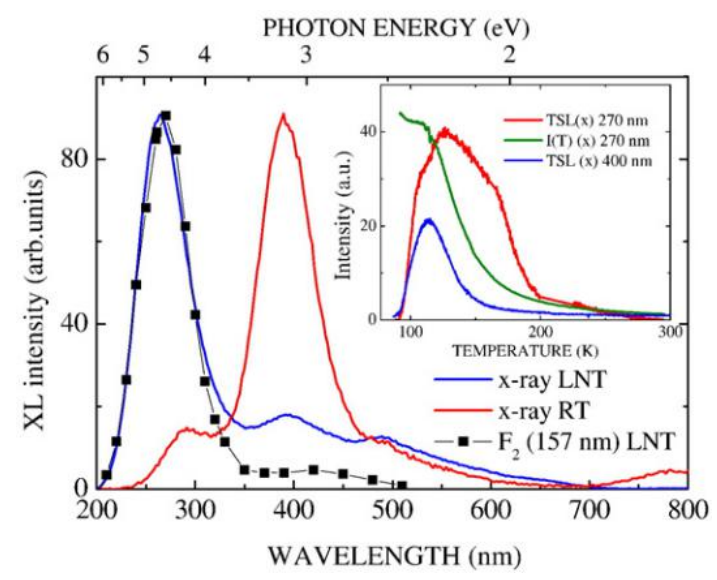

Fig. 11. Luminescence spectra of P-doped core of silica fiber preformed under excitation of $\mathrm{F}_{2}$ laser $(157 \mathrm{~nm})$ and $\mathrm{x}$-ray at $293 \mathrm{~K}$ and $80 \mathrm{~K}$. The spectra are measured differently and so were scaled so as to fit both on the same graph. Insert - thermally stimulated luminescence for different bands after $\mathrm{x}$-ray-as well as the temperature dependence of the x-ray-created $270 \mathrm{~nm}$ band, which is almost certainly due to self-trapped holes; see [13-16] and discussion at the end of Section 3.1. No TSL was detected after ArF laser irradiation of annealed at $700 \mathrm{C}$ samples.

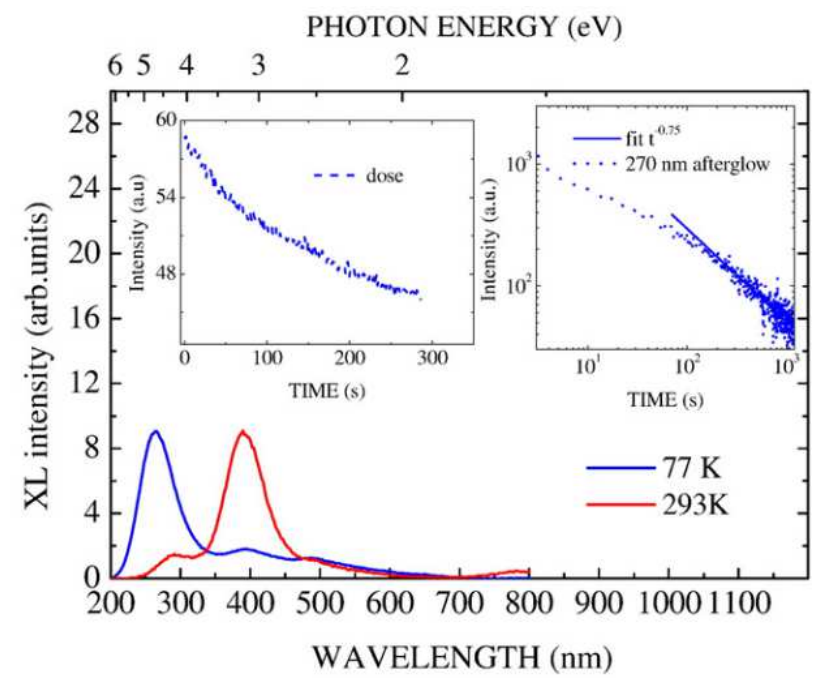

Fig. 12. X-ray excited luminescence spectra of silica with phosphorus at $293 \mathrm{~K}$ and $80 \mathrm{~K}$. Left insert: Dose dependence during X-ray irradiation. Right insert: afterglow kinetics curve of Pdoped silica fiber preform. Line is approximation of afterglow with $\mathrm{t}^{-0.75}$.

In Fig.12, the dose dependence during $\mathrm{x}$-ray irradiation and the af-terglow kinetics curve are presented as the left and right inserts, respec-tively. The decrease of $270 \mathrm{~nm}$ luminescence intensity during irradiation shows that the corresponding luminescence center partici-pates in charge trapping and therefore the quantity of emitting centers is decreased. The afterglow shows that there is a recombination of

trapped charge carriers. The kinetics could be approximated by the law $\mathrm{t}^{-0.75}$ in the end of afterglow. This may be explained by tunneling

recombination of closest pairs of electrons and holes. It is known [17] that tunneling recombination possesses characteristic $\mathrm{t}^{-1}$ law. Our value $\mathrm{t}^{-0.75}$ is close to that. N.B. The abscissa recapitulates that of Fig. 11.

Our electron spin resonance (ESR) spectra of P-doped core of a silica fiber preform are shown in Fig.13. This sample was initially annealed at $1000 \mathrm{~K}$, then cooled to $77 \mathrm{~K}$, irradiated with $\mathrm{x}$-rays, and an ESR spectrum was recorded. Subsequently, the sample was heated to $293 \mathrm{~K}$ and kept at that temperature $20 \mathrm{~h}$, then we recorded the spectrum again. The in-sert on the right shows dose dependence of signal intensity at $77 \mathrm{~K}$. The insert on left shows the DPPH spectrum (reference materials with $g=2$ ). The interpretation of the obtained ESR spectra is based on literature data. Specially, the spectrum of $293 \mathrm{~K}$ at the top corresponds to the well-known doublet signal of the Phosphorus Oxygen Hole Center (POHC)

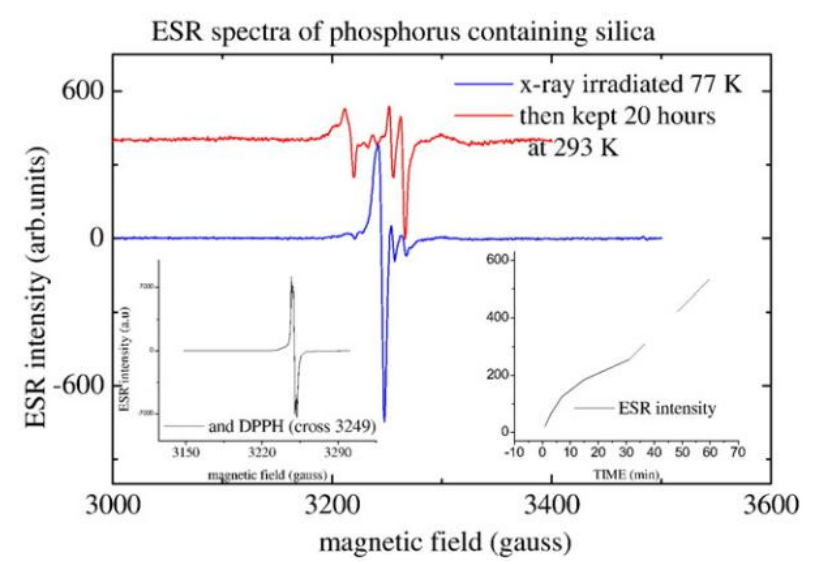

Fig. 13. Study of electron spin resonance (ESR) spectra of P-doped core of silica fiber preform subjected to $\mathrm{x}$-ray irradiation. (No signal is seen before irradiation.) The irradiated sample was initially annealed at $1000 \mathrm{~K}$, then cooled to $77 \mathrm{~K}$ and measured ESR spectrum (blue curve). Then the sample was heated to $293 \mathrm{~K}$ and kept at that temperature $20 \mathrm{~h}$. Insert on right shows dose dependence of signal intensity at $77 \mathrm{~K}$ as a function of time. Insert on left shows DPPH spectrum (reference materials with $\mathrm{g}=2$ ). 
[1] where, in the present case, the weak off-center peak is due to silicon $E^{\prime}$ centers (similar to fig. 2 insert of [16]). The spectrum obtained by x-ray irradiation at $77 \mathrm{~K}$ also includes weak POHC and E' signals. However, the principle $77 \mathrm{~K}$ spectrum (blue at bottom) corresponds well with self-trapped holes (STHs) created at that temperature (see [13-16]). During heating toward room temperature those STHs are liberated as free holes (see last paragraph of Section 3.1, above), which in the pres-ent case combine with $\mathrm{P}_{2} \mathrm{O}_{5}$ units in silica glass, thus creating the POHCs (i.e., 2-cordinated phosphorus atoms that trap a hole) see [1]. In addi-tion, some liberated holes recombine with trapped electrons to create TSL peaks (Fig.11) and afterglow (Fig.12, right insert).

In Fig.14 the influence of ArF laser irradiation on our ESR samples is shown. The sample was initially annealed at $1000 \mathrm{~K}$, then cooled to $77 \mathrm{~K}$, irradiated with pulses of ArF laser, and finally recorded as an ESR spec-trum. Then the sample was again annealed at $1000 \mathrm{~K}$, cooled to $293 \mathrm{~K}$, irradiated with pulses of ArF laser, and finally measured again by ESR. The insert on right of Fig. 14 shows the ESR spectrum of an E'-center in the ArF irradiated cladding (i.e., part of sample without phosphorus). In the case of irradiation with photons of an ArF laser, contrary to the case of x-ray irradiation, a $\mathrm{POHC}$ signal is also created at $77 \mathrm{~K}$, whereas irradiation with pulses of $\mathrm{ArF}$ laser at $293 \mathrm{~K}$ also creates a POHC signal.

\section{Discussion}

We have observed two main luminescence bands. One is in the UV range with maximum at about $270 \mathrm{~nm}$ and a blue, and another at $400 \mathrm{~nm}$. Both bands are related to the presence of phosphorus in silica glass. We did not find mutual influence of the corresponding lumines-cence centers. Therefore, these centers are attributed to different struc-tural defects in silica-glass-containing phosphorus. The slow decay of the blue band is about $6 \mathrm{~ms}$, little depending on temperature up to $500 \mathrm{~K}$. Beside the slow component, a fast component of blue-band decay is also observed in Fig.4. The time-resolved PL band for the fast component is situated at $400 \mathrm{~nm}$ as well. However, it is broader; see Fig.4. Thermal quenching of blue luminescence takes place above $500 \mathrm{~K}$ with activation energy $\mathrm{E}=0.5 \mathrm{eV}$ and frequency factor $\mathrm{f} \sim 10^{9} \mathrm{~s}^{-1}$ Fig.6. Thermal dependences of PL (Fig.6) show good corre-spondence between PL intensity and the slow decay time constant. This could mean intra-center quenching of the blue luminescence cen-ter. Comparison of PL thermal dependences with that of the ESR-studied self-trapped holes in silica glass [13-16] shows that PL-center quenching does not directly correlate with annealing of radiation in-duced $\mathrm{P}_{1}$ centers (radiation induced holes trapped on a three-coordinat-ed phosphorous ions) [1]. However, PL thermal dependence is close to

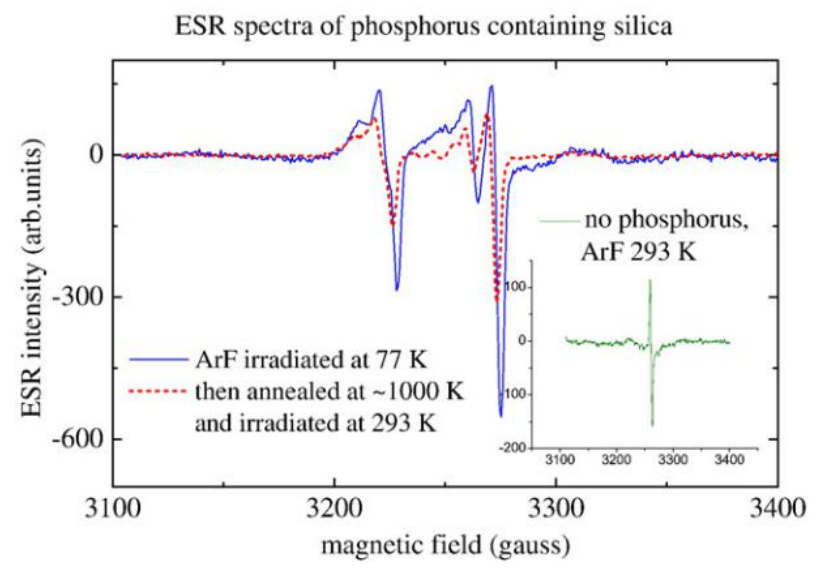

Fig. 14. ESR spectra of P-doped core of silica fiber preform. Sample was initially annealed at $1000 \mathrm{~K}$, then cooled to $77 \mathrm{~K}$, irradiated with pulses of ArF laser and measured ESR spectrum. Then the sample was again annealed at $1000 \mathrm{~K}$ cooled to $293 \mathrm{~K}$ and irradiated with pulses of ArF laser, then measured spectrum. Insert on right shows ESR spectrum of ArF irradiated cladding (part of sample without phosphorus). that of the $\mathrm{P}_{2}$ center (a 4-coordinated $\mathrm{P}$ that has captured an electron [1]) and $\mathrm{P}_{4}$ centers (2-cordinated $\mathrm{P}$ that has trapped an electron [11]). However, there are remarkable differences.

The case of KCl-P crystal [6], where blue luminescence possessing decay with $\mathrm{T}=2 \mathrm{~ms}$ (also $\mathrm{T}=90 \mathrm{~ms}$ was detected there as well), was studied here in detail, and the molecular center O-P-O (i.e., $\mathrm{P}_{4}$ [1]) was deduced. That could also be an argument in favor of such a center being created in silica glass with phosphorus, where the electronic tran-sitions are certainly triplet-singlet, as it was interpreted in [5]. In [6] the KCl-P crystal, the natural triplet state and the allowance of the triplet singlet transition by spin-orbit coupling was ascribed to states of oxy-gen. P-doped silica is ascribed to a triplet-singlet transition, and the fast decay of the blue band is ascribed to singlet-singlet transition [3]. Similar positions of time-resolved bands exhibit low-value singlet-trip-let splitting of the excited state of the center. That means that the corre-sponding wave function is spread in space.

Another known case of two-fold coordinated centers in silica is SiODC(II) and GeODC(II). For the latter center, a remarkable character-istic is the high value of singlet-triplet splitting of corresponding excited states [18]. The triplet-singlet band is situated at $450 \mathrm{~nm}(2.6 \mathrm{eV})$ for SiODC(II) and at 395 $\mathrm{nm}(3.1 \mathrm{eV})$ for GeODC(II). The singlet-singlet PL bands are situated at 280 $\mathrm{nm}(4.4 \mathrm{eV})$ and $290 \mathrm{~nm}(4.3 \mathrm{eV})$ correspond-ingly. So, the singlet-triplet splitting of excited states is about $1.8 \mathrm{eV}$ and $1.2 \mathrm{eV}$. A large singlet-triplet splitting corresponds to a wave function localized in small space. So, in the case of the P-related two-fold coordi-nated center in silica, termed $\mathrm{P}_{4}$ if it had captured a hole, it is different and corresponds better to two-fold coordinated center in KCl-P [6]. There, the wave function of the excited state is spread in space, and the spin-orbit coupling constant allows a triplet-singlet transition relat-ed to oxygen atoms, whereas in the cases of SiODC(II) and GeODC(II) the singlet-singlet transition [18] is related to $\mathrm{Si}$ or $\mathrm{Ge}$.

The previous cases include a quartz crystal with phosphorus and a phosphosilicate glass, where blue luminescence related to phosphorus was not observed. This could be explained by presence of water and/ or $\mathrm{OH}$ groups in these materials [3]. However, an actual sample of silica with phosphorus was found to be practically free of such contamination.

The intensity of UV luminescence of P-doped silica is also seen both under low intensity excitation such as deuterium discharge light source and excimer lasers. Previously we had observed the UV band in different phosphate crystals and glasses (see e.g. [2,3]). The UV band decay kinet-ics is fast ( $30 \mathrm{~ns})$, whereas there is also a slower component in the $\mu \mathrm{s}$ range of time. This situation takes place in all the cases. However, there are differences depending on excitation conditions in different materials.

Possibly, such UV luminescence is a general property of orthophosphates. Observation of the band at $210 \mathrm{~nm}$ after soldering [3] by use of orthophosphate acid is an argument for that. In the case of the $\mathrm{ScPO}_{4}$ crystal, the UV band at $210 \mathrm{~nm}$ possesses a fast component (30 ns) and slow components $(\sim 10 \mu \mathrm{s})$, which were interpreted as singlet-sin-glet and tripletsinglet transitions of the self-trapped exciton [2,4]. The STE in $\mathrm{ScPO}_{4}$ is ascribed to the $\mathrm{PO}_{4}^{3}{ }^{-}$molecular ion. In the case of crystal-line quartz doped with phosphorus it was found that this UV band con-sists of two components of decay ( $\sim 30 \mathrm{~ns}$ and $\sim 10 \mu \mathrm{s}$, see Ref. [3]). However, the UV band in quartz is situated at $\sim 250 \mathrm{~nm}$, and was as-cribed to $\mathrm{PO}^{3} 4^{-}$molecular ion as well. Presumably different surround-ings of $\mathrm{PO}^{3} 4^{-}$molecular ion in the quartz and orthophosphate of scandium explain the differences in band position. Similar interpreta-tions of the UV band in other phosphates have been made [2-4]. So, in the actual case, the UV band in silica glass with phosphorus could be ex-plained in a similar way. That is, based on observation of two compo-nents of decay, one fast and one slow, in the mentioned materials. The data presented in Fig. 9 are interpreted as singlet-triplet splitting of the excited state of the center with a value of splitting equal $0.29 \mathrm{eV}$. This is remarkably higher than that of the blue luminescence center.

The UV band thermal dependences are similar to the presently stud-ied silica glass with phosphorus and the previously studied quartz 
crystal doped with phosphorus [3], Fig.10. The decay in the crystal is faster $(15 \mathrm{~ns})$ at $8 \mathrm{~K}$ whereas in silica glass with $\mathrm{P}$ decay it is about $30 \mathrm{~ns}$.

We did not find a correlation between the center of the UV and that of the blue luminescence, with the exception that both are due to pres-ence of phosphorus. This could be explained as due to the multivalent property of phosphorus-related defects in various matrices. Phosphorus takes on a variety of valence states in different oxides doped with phos-phorus. The phosphate ion $\mathrm{PO}_{3}^{3}{ }^{-}$, the orthophosphate ion $\mathrm{PO}_{4}^{3} 4^{-}$, and the pyrophosphate ion $\mathrm{P}_{2} \mathrm{O}_{7}^{4}$ are among them [1]. These ions determine different phenomena in P-doped materials. It is clear that the $\mathrm{PO}_{4}^{3}{ }^{-}$complex is the main structural element in silicon dioxide doped with phosphorus below $600 \mathrm{~K}$ [1]. The similarities of the properties of the UV band in silica glass and that in $\mathrm{ScPO}_{4}$ suggest that the UV band in all phosphorus-containing materials may be dominated by the $\mathrm{PO}^{3} 4^{-}$complex. However, the blue band is also ascribed to this element [5]. Thus the problem needs further investigations. Two slightly differing bands are observed in thermally stimulated luminescence after $\mathrm{x}$-ray ir-radiation at LNT and RT of silica glass with phosphorus Fig.11. However, there is some difference in TSL curves for UV and blue luminescence supporting the absence of mutual dependence of these centers. De-crease of the UV band with a dose of irradiation implies the destruction of the luminescence center, presumably by trapping of a charge carrier. The afterglow (Fig. 12) witnesses the existence of a recombination pro-cess of this trapped charge with carriers of another sign.

\section{Conclusions}

UV luminescence is provided by a center existing in as-received materials and ascribed to the states related to $\mathrm{PO}_{4}^{3}{ }^{-}$ion based on the similarity of this UV luminescence with STE luminescence in $\mathrm{ScPO}_{4}$. The blue luminescence is negatively polarized when excited with polarized light. Upon warming, both luminescence bands appear in recombination of liberated self-trapped holes with the electron trapped on differing phosphorus related structures. One is in the UV lumines-cence center and the other for blue luminescence center. The first is as-cribed to $\mathrm{PO}^{3} 4^{-}$and the second is likely twofold coordinated phosphorus (O-P-O). We did not find luminescence due to electron recombination with $\mathrm{POHC}$.

\section{Acknowledgements}

This work is supported by Material Science program IMIS2 of Latvia.

\section{References}

[1] D.L. Griscom, E.J. Friebele, K.J. Long, J.W. Fleming, J. Appl. Phys. 54 (1983) 3743

[2] A.N. Trukhin, K. Smits, J.L. Jansons, L.A. Boatner, J. Phys. Condens. Matter 25 (2013) 385502 (6pp).

[3] A.N. Trukhin, K. Smits, J. Jansons, D. Berzins, G. Chikvaidze, D.L. Griscom, AIP Confer-ence Proceedings, 1624, 2014154.

[4] A.N. Trukhin, L.A. Boatner, Proceeding of the 13 IC Defect in Insulating Crystals, 239241, Material Science Forum, Wake Forest University, 1996573

[5] G. Origlio, F. Messina, M. Cannas, R. Boscaino, S. Girard, A. Boukenter, Y. Ouerdane, Phys. Rev. B 80 (2009) 205208.

[6] S.J. Hunter, K.W. Hipps, A.H. Francis, Chem. Phys. 39 (1979) 209-220.

[7] K.M. Golant, in: G. Pacchioni, L. Skuja, D.L. Griscom (Eds.), Defects in $\mathrm{SiO}_{2}$ and Related Dielectrics: Science and Technology, Kluwer Academic, London 2000, p. 427.

[8] I. Godmanis, A. Truhins, K. Hubners, Phys.Stat. Sol. (b) 116 (1983) 279.

[9] Y.A. Toyozawa, Techn. Report. ISSP A1 (1964) 119-168.

[10] A.N. Trukhin, J. Non-Cryst. Solids 149 (1992) 32

[11] L. B. Glebov, (Ph.D thesis), University of Leningrad (1975) 22.

[12] B.L. Gelmont, V.I. Perel, I.N. Jassievich, Sov Solid State Physics 25 (1983) 727

[13] D.L. Griscom, Phys. Rev. B 40 (1989) 4224

[14] D.L. Griscom, J. Non-Crystalline Solids (1992) 137.

[15] D.L. Griscom, J. Non-Crystalline Solids (2004) 139.

[16] D.L. Griscom, J. Non-Crystalline Solids (2006) 2601.

[17] A.R. Kangro, M.N. Tolstoi, I.K. Vitols, J. Lumin. 20 (1979) 349.

[18] L.N. Skuja, J. Non-Cryst. Solids 239 (1998) 16. 